Voix et Images

\title{
La Fille de Christophe Colomb : la rouerie et les rouages du texte
}

\section{Renée Leduc-Park}

Volume 5, numéro 2, hiver 1980

Yves Thériault

URI : https://id.erudit.org/iderudit/200209ar

DOI : https://doi.org/10.7202/200209ar

Aller au sommaire du numéro

Éditeur(s)

Les Presses de l'Université du Québec

ISSN

0318-9201 (imprimé)

1705-933X (numérique)

Découvrir la revue

Citer cet article

Leduc-Park, R. (1980). La Fille de Christophe Colomb : la rouerie et les rouages du texte. Voix et Images, 5(2), 319-332. https://doi.org/10.7202/200209ar d'utilisation que vous pouvez consulter en ligne.

https://apropos.erudit.org/fr/usagers/politique-dutilisation/ 


\section{La Fillo de Christophe Colomb: la rouerie et les rouages du texte.}

La Fille de Christophe Colomb' est un roman qui, grâce à la mystification mise en cuvre par le sujet, opère un nihilisme global. La réduction des valeurs s'y trouve impliquée à la fois dans les événements et dans le genre de discours employé par le narrateur. Ce texte dont le récit est divisé en deux parties s'articule sur une serie d'oppositions binaires. Ces oppositions s'annulent mutuellement par inversions successives, car il va de soi qu'un simple renversement maintiendrait les deux mèmes póles, quoiqu'en position contraire. Les "lours de roues" que constituent ces basculements répétés s'anéantissent alternativement et génèrent la force différentielle ${ }^{2}$ qui confère au lexte sa dynamique. Nous qualifions ce mécanisme de jeu (mascarade/ divertissement) dionysiaque: la destruction accomplit l'affirmation dans la nègation.

Le recll.

Si on considère d'abord l'ensemble du contenu événementiel, on se rend compte que la dichotomie temps/espace se trouve ramenée à une seule dimension grâce au chavirement l'une dans l'autre de ces deux catégories qui organisent l'existence humaine. Dans la première partie, la représentation de Christophe Colomb (23) installe le passé dans le présent car ce personnage historique vit encore au debut du récit ; il rend même visite à un "ex-matelot de la Nina" (19). Par contre, sa mort presque immédiate (26-27) élimine le rappel de celle époque antérieure. La disparition de son père inaugure chez Colombe une perte d'appartenance (85). Celle-ci parcourt alors le globe pour tenter de s'intègrer à la société contemporaine. L'énonciation au présent, caractéristique du discours du narrateur, masque la progression temporelle des événements qui marquent ce déplacement à travers l'espace. Dans cette première partie, Colombe voyage sans parvenir à son but qui est de trouver l'amilié. De fait, l'amitié n'existe plus puisqu'elle appartient au passè. Un adolescent signifie à Colombe que ce sentiment était un phénomène de I'Antiquité :

Sans avertissement, il lui flanque sur les oreilles

Quatre claques retentissantes. II est tâché comme un rentier. 
"Amitiè! Tu n'as pas honte? [...]

Amitie? C'est une des inventions pernicieuses

Des ècœurés de la Grèce antique! [...] (111)

Dans ce fragment, grâce à une serie de renversements, le passage du temps se trouve télescopé. L'adolescent se conduit comme un "rentier». De plus, celui-ci proclame la fin de l'Histoire qu'il attribue à l'attachement acruel au passé. La perpétuation des attitudes anciennes contamine le présent et garantit la perte du futur. L'amitie lui étant partout refusèe. Colombe revient à son point de départ où elle se rend finalement compte qu'il n'y a aucun avenir pour elle chez les humains et, ce faisant, renonce à s'enraciner : elle vivra au jour le jour. Dans la deuxième partie, l'heroine "s'incorpore" (133) aux anımaux. En adoptant leur point de vue, elle élimine toute perspective temporelle chronologique puisque le comportement instinctif des bètes se règle sur les cycles saisonniers. Cette circularité axée sur le déplacement de la terre autour du soleil, donc sur le mouvement dans l'espace, fait abstraction du temps linèaire et abolit le passé el le futur car l'animatité se perd dans l'instant.

Par ailleurs, les pérégrinations de Colombe permettent la représentation synchronique de personnalités et d'événements importants appartenant au plan diachronique: "De Gaulle" (42), "Tito" (60). "l'hôtel Staline" (65). Parallèlement, sont mobilisés dans l'occupation du paradis par Al Capone, les personnages d'، Hitler. Bonaparte, Pouchkine et Marat "(50). Ces avatars narratifs annulent les périodes historiques en les égalisant par contiguité. Le temps révolu se trouve infusé dans l'immédiat merveilleux de Colombe:

IIs franchissent la mer Rouge. Ils ne la tranchissent pas à la nage. II avaient si soif qu'ils l'ont bue. IIs passent a pied.

Comme les Hebreux, ils atteignent secs l'autre plage. (p. 223)

Le présent prodigieux dévalorise de la sorte l'illustre passé. Toutelois, comme l'instant n'existe que par opposition aux moments qui le précèdent et qui le suivent, la dimension temporelle s'estompe. Cette suppression réside dans le fait que l'annihilation du genre humain est annoncée à la fin du récit dans un brusque revirement du temps de l'énonciation: le présent habiluel du discours cède à un futur: "Une heure, et ils seront tous morts, hommes, femmes et enfants." (233). Cette projection du present dans le fulur affirme d'abord le temps qui, en retour, est niè par le contenu même de l'énoncè. En effet, seuls les ètres humains tiennent compte de l'avenir et comme ils sont appelés à disparaitre, le temps s'en trouve pour ainsi dire aboli.

La dimension temporelle s'abime également ou bascule dans la dimension spatiale, laquelle s'épuise à son tour par excès. Les voyages de Colombe font surgir une surabondance de lieux qui représentent les continents, pays. états el villes de toute la terre. La jeune fille touche a divers points de la planète en passant directement d'un endroil à l'autre sans que son trajet soit linéaıre. C'est ainsi que "Nice", "le Pérou", "la Grèce" (58-59). puis " l'Afrique " et "la Russie" (62-65) se font suite sur son parcours. Ce rapprochement 
de territoires séparés par de longues dıstances a pour effet de rétrécir la surface du globe. L'errance de Colombe a pour point de départ la "Biélorussie" (51), région d'exilés. Ce personnage est un "globe-trotteur " $(55,130)$. une "routière" (74, 193), libre de l'esprit de pesanteur. Le fait de se laisser guider par le hasard la remplit de joie (222). Une fois le genre humain liquidé, Colombe possedera la terre entière. Cette dilatation de son espace en opère l'atomisation par éclatement. L'anti-héroïne n'aura " de place" nulle part. Par contre, en se dispersant elle s'appropriera l'univers. Cependant, l'absence de limites détruit la notion mème d'espace, laquelle n'existe que par la différence qui constitue l'écart. Ainsi dans $L a$ FIlle de Christophe Colomb, le manque d'interstice effectué par l'élimination des frontières subsume la perte d'intervalle produite par l'abolition du passé et du futur, car le "maintenant" procède de l'aici”. Le temps et l'espace se confondent dans l'infini(té) unifiant(e) de l'informe originaire.

Cet aspect cosmique essentiellement cohésif du récit et qui relève du chaos dionysiaque, n'en exclut nullement la cohérence, car, nous l'avons mentionné plus haut, ce roman est nettement divisé en deux parties. Dans une première étape, les humains agissent sur Colombe; dans une seconde, les animaux guidés par celle-ci réagissent contre les hommes. Cette répartition est mise en relief par la numération des chapitres : chilfres romains pour la section Iraitant des ètres raisonnables et chiffres arabes pour celle concernant les bètes. Sous cette numération différente se fait également jour la contradiction mise en place dans ces deux parties opposées : le barbarisme des gens civilisés et l'humanisation des animaux. Ces inversions èvidentes s'articulent sur un renversement plus profond, c'est-á-dire sur l'installation permanente du "naturel" gráce à la destitution du *surnaturel" el au retournement du * contre-naturel ". L'univers du roman est régi par Al Capone qui, en tant qu'ltalien catholique (50), a été admis en paradis. Le bandit a usurpé la place de Dieu le Père et se fait à son tour appeler "papa". Celte Providence mortelle (fatale/humaine) se sert de Colombe pour mener à bonne fin l'extermination de l'espèce dènaturée qui peuplera son royaume céleste et pour s'emparer du domaine de Satan (95). Ouant à Colombe, elle symbolise la nature humaine dans ses deux aspects: l'animalitè, car elle ne raisonne (discute/réfléchit) pas, et le spirituel, car elle est dépourvue de " bas instincts". Ainsi, comparée aux humains dont elle possède le physique. la "sans-matière grise" (98) qui n'a reçu aucune instruction $(15,110)$ est (une) bête. En revanche, au sein de sa troupe d'animaux, elle parait "très intelligente" (201) et son humanité tranche (145). Cependant, les deux éléments: l'ange et la bête, que conjugue Colombe en son personnage se trouvent annulés puisqu'en réalité ce personnage n'est que la chose de Capone, une créature-pantin, sans " naturel".

Toutefois, les dons "surnaturels" de cette anti-héroïne angélique (elle peut voler comme un oiseau), pointent également vers une allégorie du Christ: à deux reprises on l'appelle "Christophe Colombe" $(91,117)$. "Elle marche sur le lleuve" (13), elle "fait une pèche miraculeuse" (15), sa 
croyance en l'au-delà est inébranlable $(76,105,126)$, elle pardonne à ses ennemis $(80,83)$ :

Le presbytère de Fautre est tout lézardé, tout décrépit.

Il a l'air de Jésus sur la croix, en pleine souffrance.

Elle y court comme un oiseau vers son nid.

II y a des constructions qui inspirent confiance! (126)

L'allégorie tourne à la parodie. Colombe ne parvient jamais à s'intégrer à la société des hommes quelle que soit leur idéologie. On se moque d'elle en territoire libre comme derrière le rideau-de-ter (60-68). Cet échec opere la subversion des systèmes de valeurs opposés, le christianisme et le communisme qui prèchent tous deux le grégarisme. Cette double démystification s'accompagne de plusieurs inversions egalement réductrices. De fait, avant d'essuyer tous ces revers, l'inotfensive Colombe instaure elle-même la destruction en empoisonnant son père par mégarde (23). Par ailleurs, elle se refuse à risquer sa propre extinction aux mains de ses semblables. Ce revirement de son comportement interdit toute comparaison soutenue avec le Christ. Car, le Méchant sert de protecteur à celle qui est Bonne.

Ce basculement incessant d'antinomies diverses qui travaillent le texte, se trouve dévoilé, au niveau du récit, grâce à la narration d'un événement qui abolit également l'opposition du couple "air/eau": l'ile de Manne d'ou Colombe est originaire, est mise "sens dessus dessous, comme un canot" (71) par ses habilants qui vivent maintenant "sous les flots". De plus, les Mannois ont répandu des cendres sur la partie non submergée de l'île pour retirer de l'argent des compagnies d'assurance en leur faisant croire que te pays a brúlè. Ainsi, les éléments de la nature: l'eau. l'air el le feu se combinent pour déjouer une institution sociale. les compagnies d'assurance. On assıste de la sorte à la polarisation de deux aspects de l'humanite, la nature et la culture, dont le premier prend le dessus. Dans cette optique, il est intéressant de noter que l'écusson de l'île anglaise de Man dans la mer d'Irlande, comporte la devise: "Quocunque Jeceris Stabit "3. On pourrait dè lors conjecturer qu'en decrivant le renversement de Manne, le narrateur, d'une part, remet en question la stabilité du réel, en l'occurrence la terre de Man, et que, d'autre part, il sabote la notion de "vérité immuable impliquée dans l'assertıon que constitue l'inscription emblématique. Ce dernier procédé sert de tremplin a une nouvelle pirouette subversive, auto-reférentielle cette fois, car la représentation de cette séquence événementielle chavire dans la mise en échec de la mimésis qui consiste en l'imitatıon de la réalité. En elfet, cet épisode ne reproduit pas la réalité, il la contredit et démasque du même coup le caractere illusionniste des mots dont est faite la formule héraldique. Ces inversıons simultanées de l'apparence et de la verité atteıgnent la culture à travers deux de ses expressions: l'écriture et la peinture.

Ce qui nous ramène à l'opposition “ nature/culture " dont la brouille et le brouillage se poursuivent dans la deuxième partie du roman pour aboutir au nivellement de ces deux catégories. En erfet, les animaux qui symboli- 
seraient le "naturel " $y$ assument un comportement civilisé, car Colombe les "réconcilie" tous (148); mais ce faisant, ils retombent dans la sauvagerie puisqu'il leur faut subsister. Pour ce qui est de la jeune fille qui les voit dépérir, elle doit se résoudre à employer des " méthodes criminelles " (135, 227) pour les empêcher de mourir de faim. Les humains tentent de se protéger à leur tour en lançant des bombes qui tuent des " millions " de bêtes (231) redevenues fèroces par nécessité. Celles-ci achèvent alors l'annihilation du genre humain, sous l'œil approbateur de Capone et celui, attristé mais indulgent de Colombe (232-233).

Pour résumer, le récit dépeindrait à première vue, le triomphe du Mal sur le Bien, ou de la violence sur le pacifisme. Dans la première partie, Al Capone, le Mal, l'emporte sur Dieu, le Bien; et les hommes, les méchants, agressent Colombe, la bonne; mais celle-ci est sauvée par les interventions surnafurelles d'un "matérialiste" suprême, Al Capone. Dans la deuxième partie, les bons, Colombe et ses animaux, deviennent mauvais et anéantissent les méchants, les humains, qui font alors figure de persécutés à la place de Colombe, leur ancienne victime. Cette serie d'inversions mise en branle dans $L a$ Fille de Christophe Colomb, annule les oppositions binaires et opère la réinstallation d'un seul élément, le "naturel» en détruisant la notion de "valeur " inhérente aux diverses dicholomies établies par les êtres humains. La nature rèintègre ainsi sa place en tant que nécessité de l'univers, indépendante de tout système. Car la nature n'est ni essence ni milieu; elle est brut-alité, force. "Principe générique et génésique " 4 , condition de l'espèce, elle constitue une puissance vitale et primordiale. Le culturel s'avère un supplément et non un complèment du naturel. C'est pourquoi, en mourant, les habitants de la planète sont tous acceptés au royaume surnaturel-matérialiste de Capone où ils pourront enfin se livrer à leurs pulsions, sans hypocrisie et sans se sentir culpabilisés comme ils l'étaient sous l'empire du surnaturel-spirituel. De même les animaux, en exterminant les hommes dans le but de survivre, agissent selon leurs instincts; ils ignorent la pitié, sentiment humain. Quant à Colombe qui appartient à l'espèce animale, elle n'exhibe pourtant que des vertus. Celte altitude la rend a antinaturelle" parce que perméable à l'influence d'un "surnaturel». Ce dont elle est punie: d'abord par les hommes, qui la maltraitent et la rejettent, puis par les animaux qu'elle n'arrive plus à maitriser. De la sorte, le naturel régnera enfin sur la terre et dans les cieux. Le désordre aura rétabli "l'ordre normal des choses". le désordonné ou la démesure dionysiaque qui restitue le chaos primordial.

\section{Le discours}

En dépit de la destruction globale projetée dans La Fillo de Christophe Colomb, et surtout de la violence dont font état plusieurs référents ressortissant tant au réel qu'à l'imaginaire, il ne nous parait pas interdit de ranger ce roman parmi les écrits du genre "merveilleux", car, "les événements qui se produisent ne sont nullement inquiétants " 5 . Cependant, cette classification apparemment acceptee par le narrateur: "Chers lecteurs n'oubliez pas dans vos prières/ Que je vous raconte la belle histoire de Colombe Colomb, " (195), 
se touve à son tour subvertie car les lois et normes qui régissent les sociètés représentées dans ce texte sont les mèmes qui prévalent dans l'univers réel:

Le juge Gruzelle fut élu ce soir maire de Manne-Eau.

[...

Avant de se coucher, il a pris du sel de fruit Eno.

Sa temme, elle, a tapissé sa face d'un genre de crème.

$[\ldots]$

Je sais, je sais! Ca n'empêchera pas la guerre du Vietnam

$[\ldots]$

Connaissez-vous la belle histoire de Fiam Fiam?

II mourait dans la chanson que nous chantions

Autour des feux de grève, au temps où je souffrais de diarrhée.

“Qui portera le deuil, [...] a Téton!

“Qui portera le deuil? Ce sera monsieur le curé.» (85)

En effet, cet entrelacs de la fiction et du vécu où se croisent le religieux et le scatologique dans un discours ou se résorbe le récit, relève également du genre satirique qui est fortement ancré dans l'actualité. Le narrateur signale cette contradiction: " Cette belle histoire, croyez-le ou non, fut vécue. " (195), escamotant le fait qu'il relate au présent, c'est-à-dire que la fiction jaillit au moment où il décrit, comme s'il inventait à mesure.

Toutefois, le sujet du discours ne s'attaque pas seulement à l'ordre déjà établi, mais. par un revirement de son entreprise de dévalorisation des systèmes, il pousse le nihilisme jusqu'à démolir les organismes et mouvements qui sont eux-mêmes contestataires des institutions traditionnelles. Nous nous bornerons à citer les plus probantes manifestations de ce retournement contre les puissances subversives à l'œuvre dans la société. Ainsi, la satire mine tour à tour le mariage des prêtres $(29,124-125)$, le sadisme $(81,94)$, le désarmement nucléaire $(80)$, le chómage (114), voire « La ligue pour les droits des Noirs « qui fait l'objet de tout un chapitre (174-175). L'esprit révolutionnaire n'échappe pas non plus à la caricature. Dans une séquence décrivant le désarroi de Colombe, l'énonciation confond (anéantiv déjoue/mèle) les philosophes dissidents:

Qu'a-t-elle fait que tout le monde la refuse?

Cette question se répète dans sa tête comme sur le clou

Le coup de marteau du philosophe Marcuse. (110)

L'allusion au "coup de marteau" fusionne ici dans un mème énoncé, la pensèe de Nietzsche qui se sert de cette métaphore pour illustrer la nécessité de "sonder" le phénomène de l'illusion 6 , et les théories d'un philosophe à tendance marxiste sûr de ce qu'il avance; Marcuse, lui, croit possèder la vérité en affirmant que seul un soulèvement du prolétariat aurait pu transformer le monde. Ces deux thèses syncrétisées par l'ènonciation, et qui se prononcent toutes deux pour le renversement de l'idéologie dominante. temoıgnent pourtant de visıons opposées: Nietzsche vise à exposer les utopies, les "Idoles", en particulier le socialisme qu'il abhorre?, alors que Marcuse, qui regrette l'absence de révolution travailliste, démontre son 
besoin d'illusion puisqu'il reconnaît l'impossibilité d'une telle solution ${ }^{8}$. En accolant à la formule de Nietzsche le nom de Marcuse, le sujet du discours nie tout accomplissement (achèvement/perfection) philosophique, car Nietzsche qui croyait avoir déterminé la fin de la métaphysique, a eu des successeurs colportant chacun sa theorie-vérité.

II n'en reste pas moins que dans La Fille de Christophe Colomb, c'est surtout au socialisme sous toutes ses formes que s'en prend le narrateur:

La première Jersey qu'elle voit, elle s'en empare. Tant pis! 9

Ceux qui ont soixante vaches ont volé soixante vaches!

Sans s'en apercevoir, elle raisonne comme Fourier.

Celte satire de la redistribution des biens évoquée grâce au vocable "Fourier", se répercute dans une autre séquence par le truchement du terme "fourrière" employé par une avicultrice pourtant hospitalière qui désigne ainsi de façon meprisante, le groupement forme par Colombe et sa communauté animale. L'énonciation, à travers le dispositil el la disposition des signifiants, effectue un renversement des róles: le terme ufourrière" qui signifie “ lieu de dépót d'animaux ”est appliqué à un troupeau de bétes lequel se comporte comme un phalanstere humain et qui pratique de la sorte le "fouriérisme", ce qui a pour elfet de rabaisser les partisans de ce premier stade du socialisme.

Le marxisme se trouve également ridiculisé dans les propos de Rasoir Electrique qui parodient le langage révolutionnaire. Ce personnage reproche à Colombe de ne pas être $\alpha$ in " parce que celle-ci se voudrait à l'intérieur de la société dont Rasoir, dans une nouvelle inversion, dénonce simultanément le conformisme. Les exhortations de cette dernière font basculer la satire du statu quo dans la caricature de la politique engagée:

Tu as toujours le bec tendu jusqu'aux oreilles

Et ce qui est in. C'est la moue Juliette Gréco.

Tu n'as pas l'air d'être tenue au courant, ma vieille.

II faut avoir l'air d'avoir vomi toute la journée pour être beau.

[...]

II ne laut pas être pris pour un mouton de Panurge.

II faut être pris pour un perroquet de Godard.

Chie sur les bourgeois! Dépéche! Ca urge!

Souffre, quoi! L'impérialisme Esso te mord! (90)

En plus de conclure à l'évidence de la parodie que véhicule ce fragment, il s'impose d'ouvrir ici une parenthèse afin d'attirer l'attention sur l'ènoncé : "Tu as toujours le bec fendu jusqu'aux oreilles/Et ce qui est in." qui, en enchainant avec la suite du discours, comporterait la connotation que nous lui avons attribuée plus haut, mais qui, pris isolément, marquerait la présence d'un élement négatif chez Colombe: "Tu as [...] ce qui est in." Colombe recèlerait ce qui n'existe pas ou incarnerait-(re)présenterait-“ présentifierait " l'absence puisque "in" définit également un élément locatif d'intériorité. Cette définition de Colombe - la "pleine de vide" - divulguerait la clé du 
roman, ètant donné que son histoire est celle d'un ètre issu de al'couf de Colomb": Ie texte fonctionnerait comme métaphore de l'impossible rendu possıble. Par ailleurs, pour en revenir au marxisme, l'appui des multinationales que simule le narrateur gráce au travesti du langage gauchiste qu'alfecte Rasoir Electrique, se trouve à son tour annulè dans une allusion au commerce de l'édition qui vise le capilalisme. Ce nouveau renversement figure dans une note apparaissant au bas de la page suivante et renvoyant à un auteur qui ironise: " "Le chant précédent semble être très réactionnaire. Ceux qui m'ont engagé vont être contents. (N. de l'A.)" (91) ${ }^{10}$. On remarquera que celte fois le narrateur, sujet èmetteur du discours, se dérobe et pointe vers l'auteur, dans une nouvelle version de ses interminables inversions.

Pour ce qui est d'une autre forme de socialisme, le communisme, c'est en ayant recours à l'auto-représentation que le discours l'évacue, suite à une intervention du narrateur, laquelle parodie la lutte des classes:

Les riches ne sont pas pour planter les choux tout de même.

Partageons: vous les plantez, nous on les mange.

Pas ètonnant que les pauvres soient calleux et blèmes!

Quelques vers en communiste font plaisir aux anges

Du communisme. $[\ldots](100)$

Dans le cadre de cette entreprise réductrice des idéologies, les syndicats en particulier font l'objet de la caricature, car le narrateur leur prête une mentalité qui s'apparente au fascisme dans un nouveau basculement des rôles: un agriculteur est pendu pour avoir déménagé sans "faire partie du syndicat des déménageurs" (145), un propriétaire de zoo est forcé par un "arrèté syndical" de jeter ses animaux au dépotoir (152, 174, 173). Dans un autre fragment, le sujet ridiculise l'attitude souvent dictatoriale des syndicats, dignes émules du patronat qu'ils sont censés combattre:

Rịez! Riez! Quoi? Je fais de mon mieux!

D'ailleurs, le Syndicat des tordeurs de rire

A voté une loi obligeant, veux veux pas.

Les spectateurs a manifester du plaisir

Mème si c'est ennuyant. Fais ce que dit le S.T.R. Au pas! (102)

En se retournant ainsi sur, el contre, son propre discours, le narrateur démolit le sérieux de la représentation ou de l'imitation d'une réalité-vérité. d'une valeur idéologique. Le lecteur est forcé de trouver amusant un texte qui, en se moquant de lui-méme, détruit l'illusion mimétique el perd par le fait même son caractère sécurisant. L'énonciation à l'impératif et les énoncés autoritaires imitent les contraintes auxquelles les chefs de syndicats soumettent leurs membres. En revanche, ce fragment discursif visant à détruire une institution anti-patronale. sert de pivot gènèrateur d'une séquence événementiello, cette fois, qui met en scène un syndicat à vocation contraire. Colombe et sa troupe assistent à un opéra qu'ils trouvent hilarant: 
Plaisir d'humour ne dure qu'un moment. "Que faites-vous là ?" Le Syndicat des empêcheurs de rire en rond à l'opéra*

(Syndicat affilie au Syndicat des tordeurs de rire *)

S'est manifestè sous la forme d'un sbire.

A fait son apparition, en la personne d'un policier. (185-186)

La satire au niveau du discours rebondit dans la satire au niveau du récit. Cette nouvelle pirouette caricature la propagation des syndicats et, en méme temps, les démolit: car les buts propres des deux organismes cités s'excluent l'un l'autre: le premier prétend que la représentation est solennelle comme le concept du divin, tandis que le second dont il émane pose la représentation comme divertissement léger. Le narrateur accomplit la neutralisation de ces deux perspectives opposées en établissant leur incompatibilité. laquelle résulte pourtant de leur dépendance mutuelle. Les astérisques qui figurent dans ce fragment renvoient respectivement au bas de la page ou apparaissent les sigles des syndicats en question: "S.D.E.D.R.E.R.A.L.O. et S.T.R.". Ce procédé a pour effet de parodier à son tour la façon dont certains organismes ont l'habitude de recourir à ce genre d'abréviation pour se désigner, et met sur le même pied des mouvements ou institutions à idéologies souvent totalement divergentes, tout en redoublant le fonctionnement réducteur de la satire. Tous les systemes se valent.

Dans cette optique, il serait permis de croire que le discours satirique implique une certaine mesure d'objectivité. Ce détachement de la part du sujet disparait cependant dans deux chapitres ou le narrateur de La fille de Christophe Colomb (relit) relie les aventures des personnages à ses propres sentiments en intercalant dans le récit des événements qui relèvent de sa propre " histoire". Le sujet y fait allusion á différentes grèves qui sont censées avoir eu lieu "en 1966 " et faire partie de son expérience vécue. Ces deux séquences contiguës effectuent un rapport d'équivalence entre "dockers". " marin", "ingénieurs" et "ingénieuses", et ramènent la gauche et la droite à une seule dimension:

Dieu, n'y a-t-il ici que des capitalistes et des communistes?

Dieu, tu m'as mis dans une bande de gueuleurs, de quêteurs De baveurs de slogans, de chieurs de pancartes!

Dieu, manquent-ils à ce point de cceur et d'imagination?

Dieu qu'ils m'écœurent!

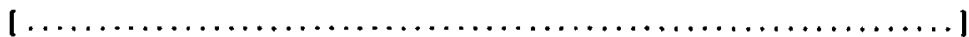

Plus fascistes que l'Église! (195-198)

Dans la première partie de cette citation, il convient de signaler que le nom de Dieu employé par le sujet de l'énonciation pour s'adresser d'abord à l'Étre Suprême, dont il prononce par le fait même l'existence, se trouve réduit à un simple explétif dans le dernier énoncé de ce fragment accusateur de l'indiffèrence divine. La Providence s'avère aussi inepte et inapte que les hommes à empêcher l'anarchie. En déclarant de la sorte ses sentiments, le 
narrateur chavire dans la subjectivité. II se montre très affecté par ces événements, tout en demeurant impartial : aucune idéologie ne trouve gràce à ses yeux. Le sujet efface ainsi la dichotomio objectivité/subjectivité en inversant constamment ces deux notions.

De fait, à l'encontre des autres romans de Ducharme, le « je m-narrateur ne remplit pas, dans La fille de Christophe Colomb, le róle de personnage principal du récit. D'une part, il s'exprime en témoin omniscient, agissant à la manière d'une caméra qui enregistre l'action d'un film. L'emploi presque exclusif du temps présent où coïncident le moment de l'énonciation et celui de l'énoncé effectue une distanciation qui est celle de la description neutre. Les paroles et les pensées des personnages se fondent et se condensent dans un discours médiatisé par le narrateur. D'autre part, cette objectivation n'est qu'apparente. Le narrateur la compromet en exprimant son opinion au sujet des sentiments cachés des personnages dont il connaît les mobiles les plus protonds (113). Ainsi, dans ce roman, les points de vue différents se réduisent à une voix narrative unique et constante, personnelle mais anonyme. Le sujet du discours se pose en tant que *je-narrateur intéressé a ses propres élucubrations en s'opposant à un "vous-narrataire" qu'il méprise et qui lui sert de "faire-valoir" (straight-man). II s'immisce également dans le récit grâce à l'énonciation de signes déictiques tels que: « D'ailleurs * (61), « Maintenant" (62), "Voici que» (63), "aujourd'hui » (64), " sans doute (65), " ma foi " (66), et par des propos commentant soit les evénements, soit son propre discours. Dans plusieurs séquences où le narrateur abuse ouvertement de la patience du lecteur, celui-ci n'en constitue pas moins l'interlocuteur du sujet de l'énonciation. Cependant, ce lecteur, en plus de servir de partenaire au "je" dans l'acte d'illocution, forme également un couple avec l'auteur, rompant ainsi l'équilibre locuteur/interlocuteur. De fait, dans La Fille de Christophe Colomb comme dans les autres romans de Ducharme, le sujet renvoie, par le truchement de notes au bas des pages, à un auteur, voire à un éditeur, ce qui gomme momentanément le narrateur en tant que générateur du récit et fait basculer le lecteur dans l'association auteur/éditeur ou production du livre. En revanche, dans ce roman, le lecteur et l'auteur ainsi réunis en dehors du texte, se retrouvent à nouveau dans le discours du narrateur, mais en tant que figurants convoqués par le je-émetteur. Celui-ci les présente d'abord comme adversaires:

Qu'est-ce que c'est que ces familiarités avec l'auteur?

Est-ce que ça va bientôt cesser?

Vais-je être obligé de vous traiter de lecteurs,

De sortir ma grosse règle et fesser? (33)

Le sujet rompt carrément avec son interlocuteur dont if méprise le statut de "lecteur" qu'il prononce inférieur à celui d'" auteur". Toutefois, cette polarisation se trouve réduite par la suite lorsque le narrateur rend le couple auteur/lecteur complice. "Sollicitant l'indulgence du lecteur, f'auteur poursuit." (175). L'énoncé qui désigne ici l'auteur comme créateur du récit, se trouve néanmoins niè par la suite dans un nouveau revirement du discours où le 
sujet affirme avoir tiré I'histoire de Colombe de son propre subconscient (195).

On assiste de la sorte à une fragmentation du sujet dont le décentrement achève une hétérogénéité englobante, car it se situe simultanément à l'intérieur et à l'extérieur du procès d'énonciation. Autrement dit, grâce à la nomination de l'auteur, le " je" du discours s'esquive au profit de celui-ci qui devient le narrateur-à-la-troisième-personne. D'autre part, ce même "je" étant la source (ou l'auteur) de ce procédé, se trouve à tirer les ficelles, ce qui le situe à la périphérie d'où il surplombe la totalité du texte et en régit le fonctionnement autant que la représentation. Ces détours par l'auteur désorientent le lecteur (le liseur). Par ailleurs, étant manipulés el désignés à la troisième personne par le “ je "-narrateur, l'auteur et le lecteur représentés deviennent à leur tour personnages; le sujet du discours leur fait jouer son rôle. Dans l'énoncé: a l'auteur poursuit", ce dernier est spécifiquement indiqué comme étant celui qui raconte. Quant au lecteur, il est ailleurs invité à inventer lui-même le récit: : Devinez la suite. Moi, je suis fatigué./ Je vais me détendre un peu de ce travail sot./ Je suis comme un avion. Je me plais dans les auteurs élevés. (128). Bien que constamment sollicité en tant qu'interlocuteur $(22,47,72,190,218)$, le dénommé lecteur n'est qu'une marionnette au méme titre que Colombe qui est le jouet d'Al Capone. Cet interlocuteuralibi sert de dispositif de raisonnement-résonnance au narrateur.

Le " je" du discours discute avec lui, mais ce n'est pas à cet interlocuteur que s'adresse son énonciation en tant que "procès d'énonciation " ". Dans La Fille de Christophe Colomb, l'objet du désir du sujet consiste en une deuxième personne, dans les deux sens de cette expression: le * fu-vous " de l'acte illocutoire et "l'Autre" qui constituent le véritable allocutaire de l'énonciation. Car, comme les autres narrateurs chez Ducharme, le sujet qui est du sexe masculin dans La Fille de Christopho Colomb, cherche á s'assimiler l'élément opposé afin d'achever la complémentarité de l'androgyne:

J'ai la bouche fermée dur de ne pouvoir croquer ton visage.

Moi qui deviens fou en t'attendant.

$[\ldots]$

Tant pis, je te verrai peut-être ici,

Assise sur mon lit de moine pénitent. (194)

Cette allocutrice apparait pour la premiere fois au chapitre XXXXIV oủ le narrateur décrit la Bulgarie que visite Colombe (61). En parodiant L'invitation au voyage de Baudelaire, dans lequel le poète fait miroiter un pays où " tout n'est qu'ordre et voluplé", c'est-à-dire agréable, alors que la vie sous l'étroite dépendance soviétique est régimentée mais déplaisante, le sujet convoque celte deuxième personne sous diverses appelations: "douce amie", "mon ange". "ma grande", "ma fouine", "ma sœur", "mon enfant". Il prévient d'abord les doutes de celle-ci sur la véracité de ce qu'il raconte, puis s'alfirme: "Je sens que tu vas bientót me traiter de menteur./ Ici, ment qui dit à un fou de se laire." (62). L'énonciation assertive de ce dernier vers inverse 
les positions du locuteur et de l'allocutrice. En effet, si les habitants de la Bulgarie sont soumis à un régime totalitaire et n'ont aucun droit, leur gouvernement en retour se trouve régi par les diktats du Kremlin et privé d'autonomie: "le gouvernement seul à gouverner" y "est le seul a être gouverné". Celui qui nie cel état de choses contradictoire admet le règne absolu de la logique ou bièn s'aveugle délibérément sur le communisme. Le narrateur souligne celte aberration en renvoyant à son propre discours qui s'applique également à la situation qu'il vient de décrire. Sous l'apparence du non-sens émis se devine l'absurdité d'une réalité politique "incroyable". Cette série d'inversions simultanées gomme la distinction entre l'apparence et la vérité el confirme l'ubiquitè de l'illusion puisqu'on n'arrive jamais à (re)tenir le sens définitif des énoncés dont les signifiés en rebondissant l'un dans l'autre, échappent à toute saisie.

Dans cette optique, il n'est pas étonnant que le narrateur rende compte de la distraction de son allocutrice devant l'inanité de son discours. Après avoir accumulé les redondances, il interrompt le récit et entame un nouveau sujet de conversation: " [...] Excusez-moi si je vous ai dérangèe./ Etes-vous allée voir le dernier Bunuel?» (60). Le sujet rompt le fil du discours pour s'approp'rier l'attention de l'Autre dont l'identité féminine est révélée par l'accord des participes passés. Ces énoncés marquent également le peu d'importance qu'il attache au récil dont la narration rappelle la technique du cinèaste espagnol mentionné. Grâce à ce procédè, le “jè retombe dans l'objectivité tout en réalisant sa propre subjectivation qui consiste à assujettir la deuxième personne. L'anarchie produite par ce chavirement répété du couple subjectivité/objectivité au niveau de la satire, et par le décentrement du sujel vers celui de l'énonciation, participe du nihilisme mis en ceuvre dans La Fille de Christophe Colomb.

\section{La prosodie}

Par ailleurs, on pourrait également avancer que ce roman constutue un défi, celui de reduire l'opposition prose/versification. De prime abord, il est evident qu'il s'agit d'un discours rimé. Cependant, cette prétention de s'exprimer en vers se trouve détruite par la présence dans certains quatrains d'ènoncés qui dérogent à celte convention et introduisent l'asymétrie. De fait, ce manque d'assonance, transgresseur par rapport à l'ensemble du discours, disséminé ça et là et qui se décèle a peine, doit être sıgnalé par le narrateur:

Si j'étais moins écœurè de la vie.

Je te raconterais sans doute mieux celte belle histoire.

Quand on a envie de se suicider, mon amie,

Les vers qu'on fail on les brise à mesure. (195)

Dans ce fragment, on constate que l'absence de rime se manifeste sous le signe de l'auto-destruction. Le narrateur évoque son anéantissement personnel dans un ènoncé qui fait allusion à la démolition de l'entreprise de versification. L'auto-représentation projette l'anti-représentation: le retournement contre soi du sujet se dissıpe en chavirant dans le retour sur soi du 
discours qui, à son tour, bascule dans sa propre ruine. Dans cette perspective, on peut s'attendre à ce que la versification soit retirée des quatre derniers quatrains du texte qui décrivent l'extermination du genre humain. Ce revirement formel du discours qui marque la destruction projelée dans le récit. indique que le narrateur compose dans une certaine mesure avec les événements représentés: l'objectivité de la satire se teinte de subjectivité.

D'autre part, il est possible de détecter dans la défectuosité de la versilication, la délaite du couple son/sens. En général, le discours rimé qui vise à maintenir l'identité des sons à la fin de chaque vers, produit un écart de sens, lequel génère souvent un nouveau sens. Dans La Fille de Christophe Colomb, au contraire, la nécessité de l'assonance opère à plusieurs reprises l'écartèlement du sens. Plusieurs énoncés ne "riment à rien " et formulent le non-sens:

Plaignons celui que, à un moment, le public aima.

Les oiseaux volent. Les poissons nagent.

Plus ca va plus mes quatrains empirent.

Mais ce n'est pas de vos sales affaires.

Mêlez-vous de vos oignons et de vos cires

Qui nettoient tout en cirant. Bandes d'éphémères! $(20,37)$

Ce fragment où se réitère l'insouciance apparente du narrateur par rapport au contenu de son récit et sa désaffection vis-à-vis du lecteur, met tout le texte en abỳme (ce texte qui abime tout). Car l'écartèlement du sens permet au sujet d'affirmer que ses vers n'ont pas de sens et de mentionner la façon dont il entend grouper ses énoncés. Toutefois, ce pointage ostentatoire n'est encore une fois qu'un leurre: à plusieurs reprises, le discours se dérobe à cette règle pour rassembler les vers en alignements de cinq (169, 185-186), trois (198) et six (201) rimes. Cette nouvelle subversion de la forme défie la classification et se rallie à l'asymétrie dissimulée de la versification.

La disjonction formelle ainsi effectuée souscrit à la désorganisation sociale inscrite dans la satire de tous les systèmes et à la décomposition du sujet de l'énonciation. Le ravage complet des valeurs, dévastation prudemment déguisée à travers les avatars d'un discours s'articulant dans un mouvement rotatif, étaierait donc en le sous-(en)tendant le dénouement nihiliste du récil qui seul annonce ostensiblement la destruction.

Renée LEDUC-PARK Université de Toronto 
1. Ducharme. Rejjean, La Fille de Christophe Colomb, Paris, Gallimard, 1969, 332 p.

2. “[...] c'est dans la roue que doit ètre pensée la Selbstuborwindung. c'est en elle aussi, par voie de conséquence, qu'il faut penser son elfet, le renversement des contraires. (... En cela se trouve contenue la vertu exceptionnelle de ce qui se répète: en ce qu'il peut se “ surmonter soi-méme ", s'accomplir en s'aliénant dans une différence absolue à soi, autrement dit : en se niant et en s'annulant en soi, dans un mouvement de sortie hors de soi suivant la trajectoire d'une roue. L'histoire d'une roue est l'histoire de ce procès de répétition et d'annulation. d'annulation dans la repetition circulaire, en quoi l'on peut reconnaitre la marque de négation absolue de soi, le pathos de la plus grande distance à soi. ce que nole dans la problématique nietzschéenne le concept de "maîtrise". " Bernard Pautrat, Versions du soleil. Paris, Seuil, 1971, p. 15.

3. Traduction libre: "de quelque façon que tu me lances, je relombe toujours a l'endroit."

4. D'après un compte rendu de Heinz Weinmann: a Edgar Morin: l'ordre du désordre «, sur un ouvrage d'Edgar Morin. La Móthode, 1, La nature de la nature, Paris, Seuil, 1977, 38 ? pp., dans Lo Devoir. 18 mars 1978, p. 87.

5. Tzvétan Todorov, Introduction à la littérature fantastique, Paris, Seuil, 1970, p. 180.

6. Frédéric Nietzsche, Le crépuscule des idoles, "Avant-propos «, Denoël/Gonthier, Mercure de France, 1899, p. 8.

7. Nietzsche, Humain trop humain II, lbid., p. 124-125.

8. D'aprés Wolle Moore. The Critical Spirit, Essays in Honor of Herbert Marcuse, Boston, Beacon Press, 1967, p. 377, 385.

9. Jeu de mots: "pie de vache/tant pis".

10. Le mot "engagé * est souligné dans le texte.

11. Emile Benveniste, "L'appareil formel de l'enonciation » dans Langages, 17 (mars 1970), p. 12-13. 